\title{
The maximum power point tracking based-control system for small-scale wind turbine using fuzzy logic
}

\author{
Quang-Vi Ngo ${ }^{1}$, Chai $\mathrm{Yi}^{2}$, Trong-Thang Nguyen ${ }^{3}$ \\ ${ }^{1}$ Faculty of Electronic and Electrical Engineering, Haiphong Private University, Vietnam \\ ${ }^{1,2}$ College of Automation, Chongqing University, China \\ ${ }^{3}$ Faculty of Electrical and Electronic Engineering, Thuyloi University, Vietnam
}

\begin{tabular}{l} 
Article Info \\
\hline Article history: \\
Received Dec 22, 2019 \\
Revised Mar 25, 2020 \\
Accepted Mar 29, 2020 \\
\hline Keywords: \\
Fuzzy logic \\
Hill climbings searching \\
MPPT \\
SSWT \\
Wind turbine
\end{tabular}

\begin{abstract}
This paper presents the research on small-scale wind turbine systems based on the Maximum Power Point Tracking (MPPT) algorithm. Then propose a new structure of a small-scale wind turbine system to simplify the structure of the system, making the system highly practical. This paper also presented an MPPT-Fuzzy controller design and proposed a control system using the wind speed sensor for small-scale wind turbines. Systems are simulated using Matlab/Simulink software to evaluate the feasibility of the proposed controller. As a result, the system with the MPPT-Fuzzy controller has much better quality than the traditional control system.
\end{abstract}

\section{Corresponding Author:}

Trong-Thang Nguyen,

Faculty of Electrical and Electronic,

Thuyloi University,

175 Tay Son, Dong Da, Hanoi, Vietnam.

Email: nguyentrongthang@tlu.edu.vn

\section{INTRODUCTION}

In recent years, the sustainable energy source has been a topic of great interest and research. In particular, the source of wind energy is one hot topic [1-4]. Medium and large wind turbines have been installed in the mainland and offshore. However, in isolated areas and urban areas, the medium and large wind turbines cannot be installed. Therefore, the Small-Scale Wind Turbine (SSWT) has been applied to take advantage of these regions [5-7]. The direct-drive SSWT using a permanent magnet synchronous generator (PMSG) has advantages such as eliminating gearbox, eliminating noise and without the excitation system. Thus, it makes less maintenance of the system and reduces the system cost. The common feature of wind energy systems is controlling to reach the maximum power point of the output energy. Therefore, this paper presents and analyzes the algorithmic structures related to MPPT, then proposes the MPPT based-system structure for SSWT.

There are some MPPT algorithms such as the following:

- The tip speed ratio (TSR) algorithm requires an anemometer. The characteristics of this algorithm are simple, fast response but not high accuracy. The turbulence of the wind speed makes the output power unstable [8-10].

- The power signal feedback (PSF) algorithm requires the information of the maximum power curve of a wind turbine $[9,11]$. This algorithm does not use an anemometer but uses electrical sensors to measure the input signal.

- Based on the optimal torque (OT) algorithm, the torque of the generator is controlled according to the reference curve of optimum torque from the maximum power of the wind turbine at the corresponding 
time of the wind speed [9, 11-13]. This algorithm requires information about the air density and the mechanical parameters of the turbine. This method is simple, fast but less effective than TSR method because the wind speed is not directly measured, means that the changes of wind are not reflected immediately on the set signal.

To overcome the disadvantages of the above algorithms, the hill climb search (HCS) algorithm or perturbation and observation (P\&O) algorithm are proposed. This algorithm is the optimal technique for finding optimal power-point. This method uses the sensors to monitor the direct current and the direct voltage $[8,14]$ then the step-size is generated by comparison between the two signals. This method can also use a tachometer of the generator for measuring the power and generating the step-size [15, 16]. This method is low cost, high reliability, and stability. However, the drawback of this method is fixed step-size, while the wind speed often changes. Therefore, when the wind speed variables change rapidly, the maximum power point cannot be found. Additionally, choosing an optimal step-size is not an easy task when the system has a large inertia.

The traditional MPPT algorithm is improved in studies [8, 11, 14, 17]. These methods are a combination of conventional and nonlinear algorithms. It can change quickly the step-size to response to the output change of the algorithm. However, these improved algorithms have to offer many cases for comparison, so the program memory of the algorithm is large and the processing speed of the controller is decreased. Some studies [18]-[20] proposed a combination of conventional algorithms and the neural network. The advantages of the neural network are that the ability to learn is quick and it doesn't need the object model. However, the downside of this method is the slow ability to calculate the step-size, so it is difficult to apply in practice.

The fuzzy logic algorithm has advantages such as able to apply the knowledge of the expert to the wind turbine system, fast response algorithm, high flexible and don't need to know the math equation of wind turbine system $[5,16,21]$. Therefore, this study will design an MPPT controller using fuzzy logic for SSWT. The proposed algorithm will take two signals to create step-size: the power of the wind turbine and the speed of the generator. These two signals are compared to create step-size for the controller. Then, the fuzzy controller gives the optimal step for the system. This proposed algorithm will compare with the conventional algorithm HCS through the simulation software Matlab/Simulink to prove the advantages of the proposed MPPT-fuzzy controller.

\section{THE SYSTEM STRUCTURE OF SSWT}

\subsection{The wind turbine model}

The power of the wind turbine is calculated by (1) [8]:

$$
P_{t}=C_{p}(\lambda, \beta) \frac{\rho A}{2} V^{3}
$$

where, $C_{p}$ is the power conversion coefficient that is as (2). Plotting of the power conversion coefficient is shown in Figure 1.

$$
C_{P}(\lambda, \beta)=b_{1}\left(\frac{b_{2}}{\lambda_{i}}-b_{3} \beta-b_{4}\right) e^{\frac{-b_{5}}{\lambda_{i}}}+b_{6} \lambda
$$

With $\frac{1}{\lambda_{\mathrm{i}}}=\frac{1}{\lambda+0.08 \beta}-\frac{0.035}{1+\beta^{3}}$

The ratio between the turbine blade speed and the wind speed is as follows:

$$
\lambda=\frac{R \omega_{t}}{V}
$$

Therefore, the output power of the turbine is as follows:

$$
P_{t}=\frac{1}{2} \pi \rho R^{2} C_{p} V^{3}
$$

The torque of a wind turbine is as follows:

$$
M_{t}=\frac{P_{t}}{\omega_{t}}
$$


The wind turbines can operate according to different control rules depending on the wind speed, the detail is shown in Figure 2.

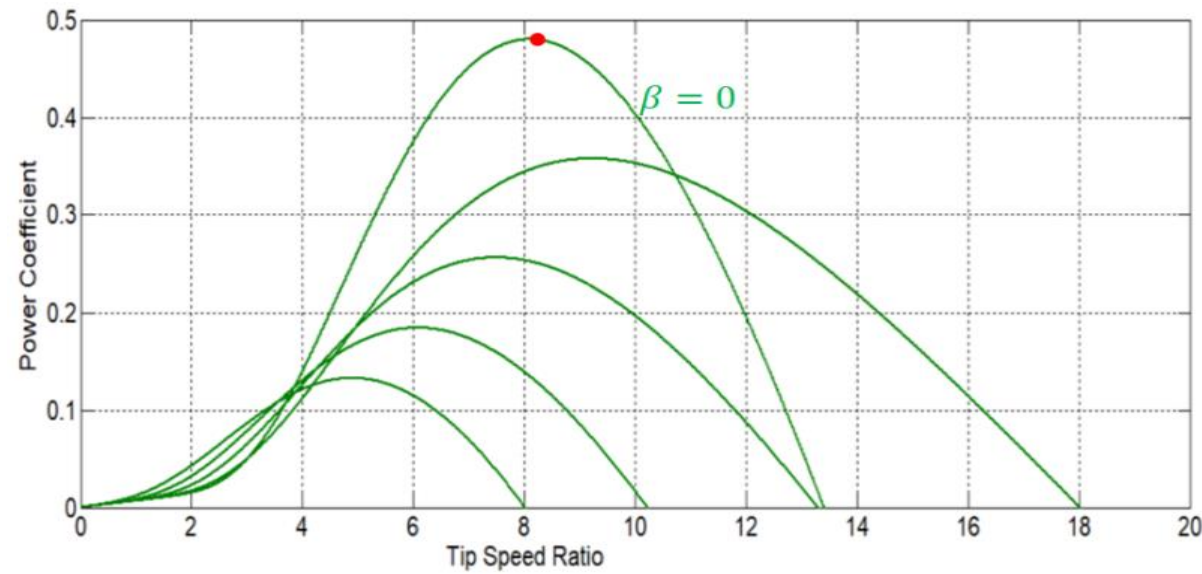

Figure 1. The power conversion coefficient $C_{P}-\lambda(\beta$ is the pitch angle)

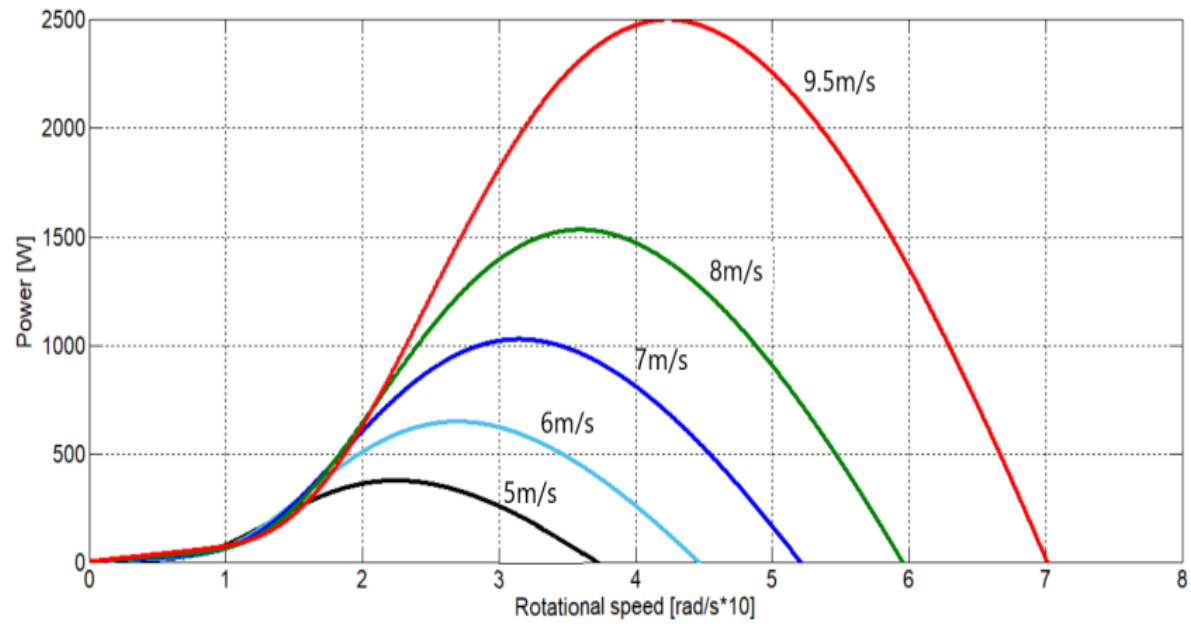

Figure 2. The power versus $\omega_{t}$ for different wind speeds

\subsection{The PMSG model}

The current and voltage equations on the coordinate system d-q [8]:

$$
\left\{\begin{array}{l}
\frac{d i_{d}^{s}}{d t}=\frac{1}{T_{d}^{s}} i_{d}^{s}+\omega_{s} \frac{L_{q}^{s}}{L_{d}^{s}} i_{q}^{s}+\frac{1}{L_{d}^{s}} u_{d}^{s} \\
\frac{d i_{q}^{s}}{d t}=-\omega_{s} \frac{L_{d}^{s}}{L_{q}^{s}} i_{d}^{s}-\frac{1}{T_{q}^{s}} i_{q}^{s}+\frac{1}{L_{q}^{s}} u_{q}^{s}-\omega_{s} \frac{\psi_{p}}{L_{q}^{s}}
\end{array}\right.
$$

where: $L_{d}^{S}$ - the stator inductance measured on vertical axis; $L_{q}^{S}-$ the stator inductance measured on horizontal axis; $\psi_{p}$ - the flux; $T_{d}^{s}, T_{q}^{s}$-the time constants of stator.

Torque equation of the generator:

$$
M_{g e n}=\frac{3}{2} P_{c}\left[\psi_{p} i_{q}^{s}+i_{d}^{s} i_{q}^{s}\left(L_{d}^{s}-L_{q}^{s}\right)\right]
$$

$\mathrm{P}_{\mathrm{c}}$ is the number of polar pairs 


\subsection{The MPPT systems for the SSWT}

\section{a. The MPPT system with sensors}

The system shown in Figure 3 has been used extensively in medium and large wind turbine systems $[10,22]$. This structure uses a sensor that measures the wind speed, sensors for measuring the direct and alternating current, and the direct voltage. The system uses 6 IGBTs on the machine side to ensure the power in the low-speed areas is exploited to the maximum capacity [23, 24]. This method is often combined with the pitch control system to adjust the pitch angle in case the wind speed is greater than the norm. The cost of the system is high, but in return, the system is highly reliable and identifying the optimal power-point.

b. The sensorless MPPT system

This scheme shown in Figure 4 is widely used in SSWT systems 5, 8, 14, 17, 19, 25]. The advantage of this method is that it does not use an anemometer, only uses electric sensors so the system has a long life and reliable operation $[23,24]$.

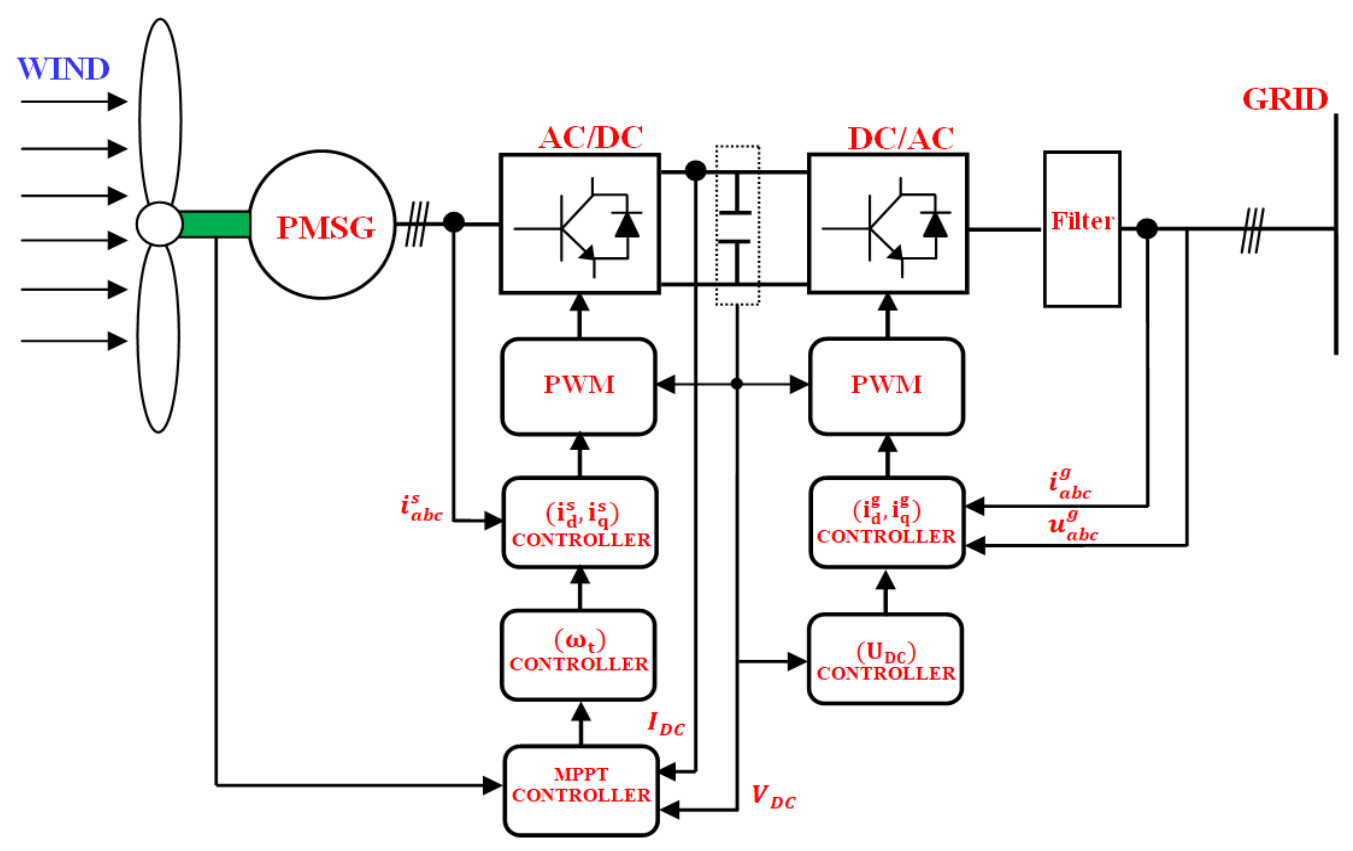

Figure 3. MPPT structure with a wind speed sensor

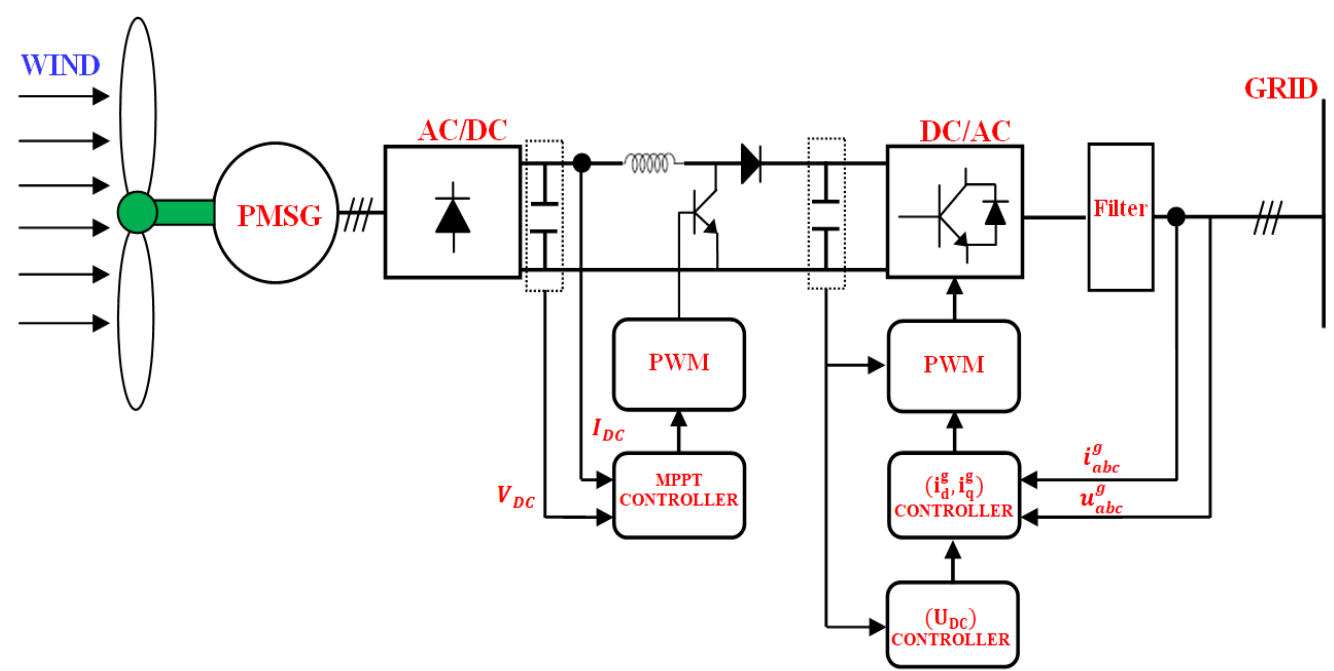

Figure 4. The sensorless MPPT structure for SSWT 


\section{DESIGNING THE MPPT CONTROLLER FOR SSWT}

\subsection{The SSWT structure using Direct - Drive PMSG}

The proposed structure is shown in Figure 5. The authors used wind speed meters and output transformers to ensure grid connection for the $380 \mathrm{~V} \mathrm{AC}$ system. The structure has eliminated the current sensor and voltage sensor because the SSWT system has guaranteed stability with different wind speeds. The system uses two signals such as the wind turbine power and the speed of the generator as the inputsignals for the MPPT. This structure also eliminates the gearbox system, so the space of the wind turbine is wider, the weight of the system is reduced and the installation process is actually easier. Therefore, this system is very suitable to install the turbine in isolated areas or urban areas. The proposed system also has the control section for the pitch angle so the SSWT system can operate in a wide range of wind speed with different regions. This structure has eliminated traditional elements such as a dump load, a passive pitch controller and a furling system.

\subsection{The control strategy of the SSWT system}

Assuming that ignoring the losses in the system, the relationship curves between the turbine power $\left(P_{t}\right)$ and the generator speed $\left(\omega_{t}=\omega_{s}\right)$ are shown in Figure 6. With a definite value of wind speed, the task of the MPPT controller is controlling the speed of the generator in order the power taken from the wind to the grid is maximum. For example, if the wind speed is $V_{1}$, the generator speed is $\omega_{t 1}$, the power that the system transmits to the grid is point $A$, the MPPT controller will control the system to reach the speed of $\omega_{\mathrm{t} 2}$ corresponding to the transmitted power reach the maximum at point $B$. Similarly, if the wind speed increases to $V_{2}, V_{3}$, the MPP controller will control the system reach the speed of $\omega_{t 3}$ (point F), $\omega_{t 4}$ (point D) respectively to achieve maximum power.

\subsection{Designing the MPPT-Fuzzy controller for SSWT}

The fuzzy-controller is based on the human experience through a set of experiential design rules. It also does not require the exact mathematical model of the control object. Design of a fuzzy controller has the three steps:

- Fuzzification: Converting the input variable from the real value to the fuzzy value.

- Setting the fuzzy logic rules "if ...then"

- Defuzzification: Converting output value from the fuzzy value to the real value for controlling the object.

Fuzzification of the input and output are shown as Figure 7, including linguistic variables: Decrease Big (DB), Decrease Small (DS), Zero (ZE), Increase Small (IS), and Increase Big (IB). The control rules are setup through expert knowledge and experience of the authors. There are 25 control rules which are shown in Table 1.

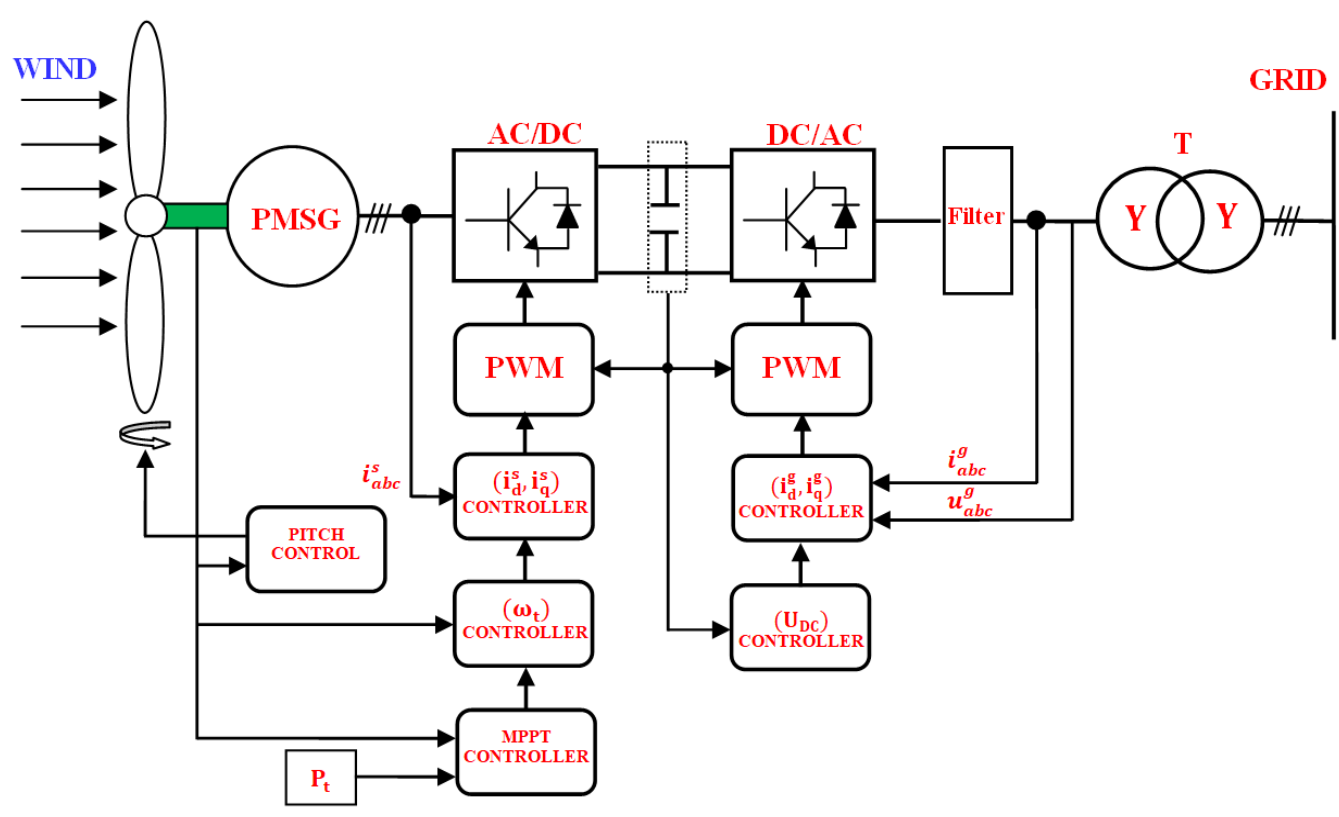

Figure 5. The proposed SSWT system structure 


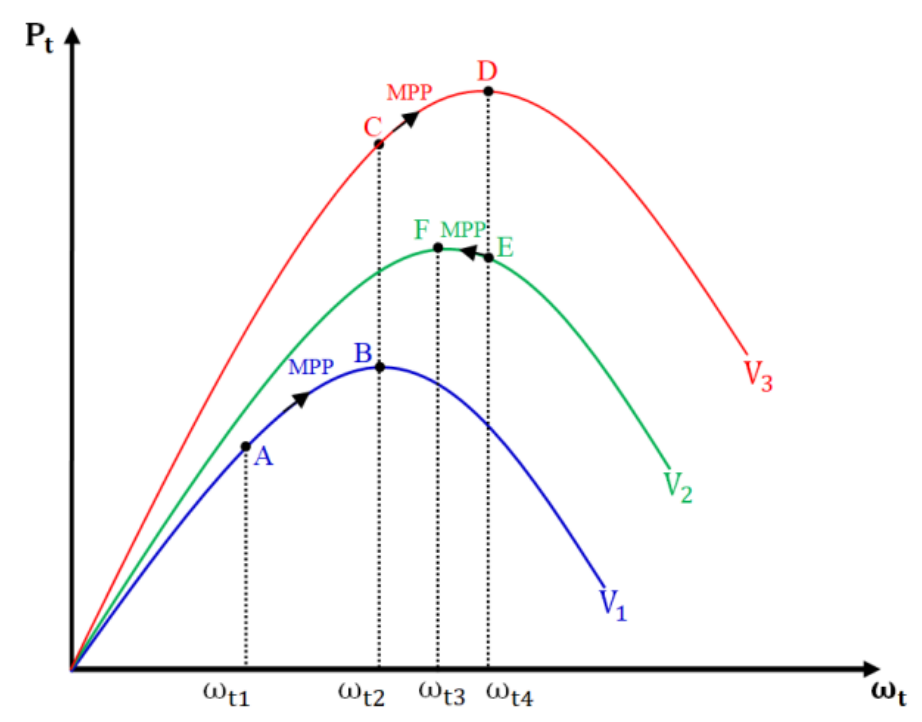

Figure 6. The relationship between the turbine power, the generator speed, and the wind speed

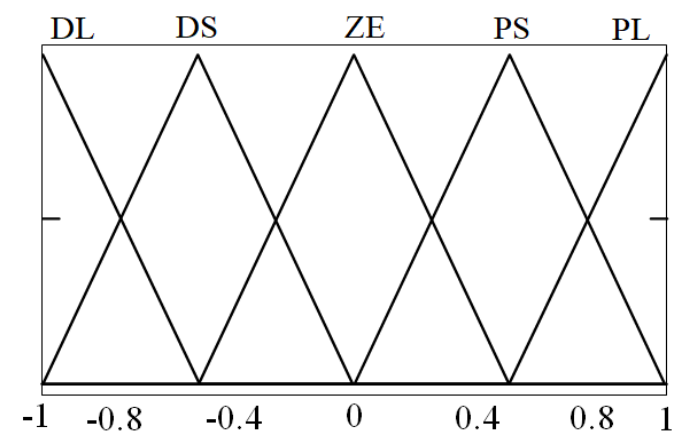

Figure 7. The linguistic variables

Table 1. The rule base of Fuzzy controller

\begin{tabular}{ccccccc}
\hline \multicolumn{2}{c}{$\Delta \omega_{\mathrm{t}}^{\text {ref }}$} & \multicolumn{5}{c}{$\Delta \mathrm{P}_{\mathrm{t}}$} \\
\cline { 3 - 7 } & & DB & DS & ZE & IS & IB \\
\hline \multirow{4}{*}{$\Delta \omega_{\mathrm{t}}$} & DB & IB & IS & ZE & DS & DB \\
& DS & IS & IS & ZE & DS & DS \\
& ZE & DB & DS & ZE & IS & IB \\
& IS & DS & DS & ZE & IS & IS \\
& IB & DB & DS & ZE & IS & IB \\
\hline
\end{tabular}

The diagram of the MPPT-Fuzzy control system is shown in Figure 8, and the Parameters of SSWT in research is shown in Table 2. The change of the output power of the wind turbine at the cycle $(\mathrm{k})$ is as follows:

$$
\Delta P_{t}[k]=P_{t}[k]-P_{t}[k-1]
$$

The change of the generator speed at the cycle $(\mathrm{k})$ is as follows:

$$
\Delta \omega_{t}[k]=\omega_{t}[k]-\omega_{t}[k-1]
$$

The optimal speed at the cycle $(\mathrm{k})$ :

$$
\omega_{t}^{r e f}[k]=\omega_{t}[k-1]+\Delta \omega_{t}^{r e f}[k]
$$


The optimal reference-speed of the controller:

$$
\omega_{t}^{n e w_{-} r e f}=\omega_{t}^{r e f}-\omega_{t}
$$

where $G_{1}, G_{2}$ and $G_{3}$ are the adaptive coefficients.

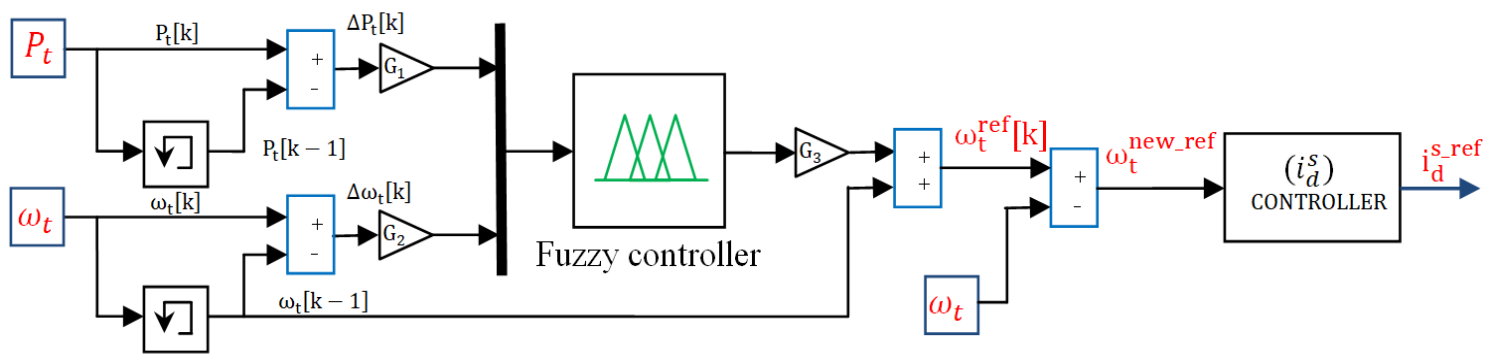

Figure 8 . The diagram of the MPPT fuzzy controller

Table 2. The Parameters of SSWT

\begin{tabular}{ccccccc}
\hline Rated power $(\mathrm{kW})$ & $\begin{array}{c}\text { Radius of } \\
\text { rotor }(\mathrm{m})\end{array}$ & $\begin{array}{c}\text { Air density } \\
\left(\mathrm{kg} / \mathrm{m}^{3}\right)\end{array}$ & $\begin{array}{c}\text { Power } \\
\text { coefficient of } \\
\text { optimal }\end{array}$ & $\begin{array}{c}\text { Tip speed ratio } \\
\text { of optimal } \\
\lambda\end{array}$ & $\mathrm{b}_{1}-\mathrm{b}_{6}$ of coefficients & $\begin{array}{c}\text { Rated wind } \\
\text { speed }(\mathrm{m} / \mathrm{s})\end{array}$ \\
\hline 2.5 & 1.8 & 1.225 & 0.48 & 8.1 & $11 ; 0.4 ; 5 ; 21 ; 0.0068$ & 9.5 \\
\hline
\end{tabular}

\section{RESULTS AND ANALYSIS}

The authors ran the system with two different MPPT controllers: HSC and Fuzzy. Figure 9 shows the wind speed. This speed ranges from the start-up wind speed to the rated wind speed. At these regions of the wind speed that MPPT controllers need to find the maximum power.

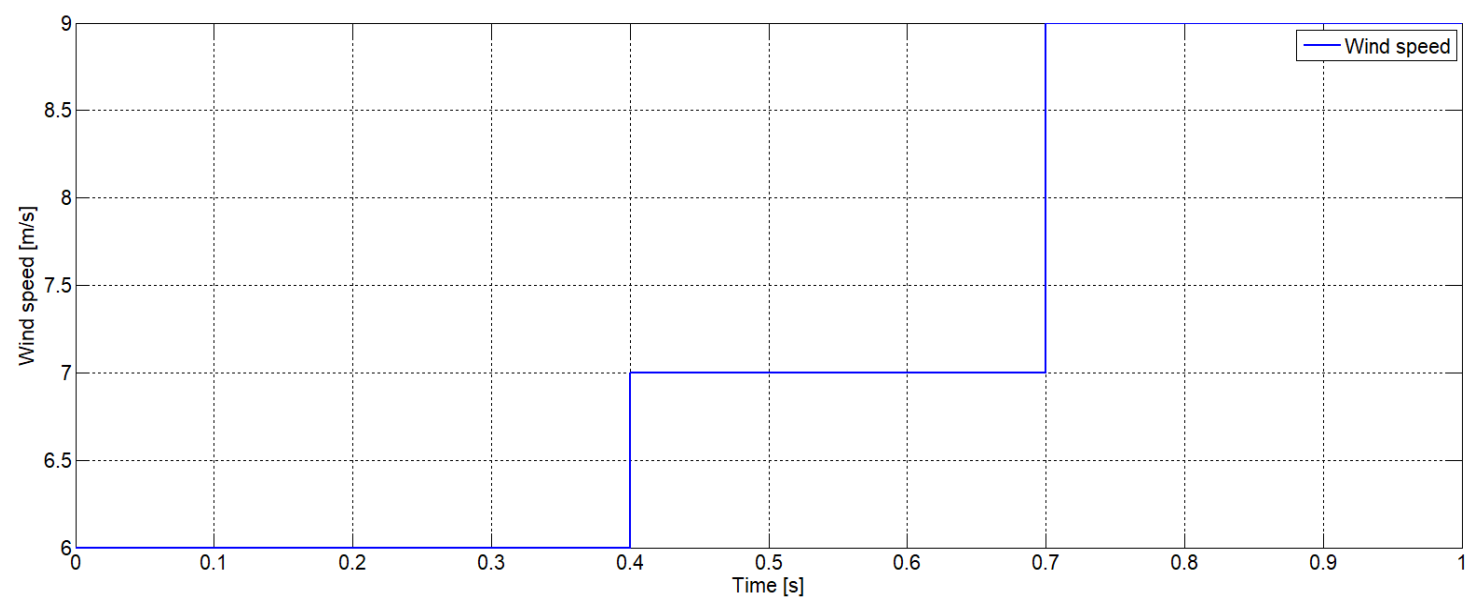

Figure 9. The wind speed

In order to ensure the maximum power, the energy conversion coefficient $C_{P}$ always follows the optimal value. The characteristic of $C_{P}$ is shown in Figure 10. The simulation results show that in the first period of $0-0.1 \mathrm{~s}$, the Fuzzy controller's power coefficient responds quickly and closely to the value of 0.48 , while the HCS controller was slower. At times $t=0.4 \mathrm{~s}$ and $0.7 \mathrm{~s}$, the wind speed changes, we see the system with the Fuzzy controller set up faster than the system with the HCS controller. 


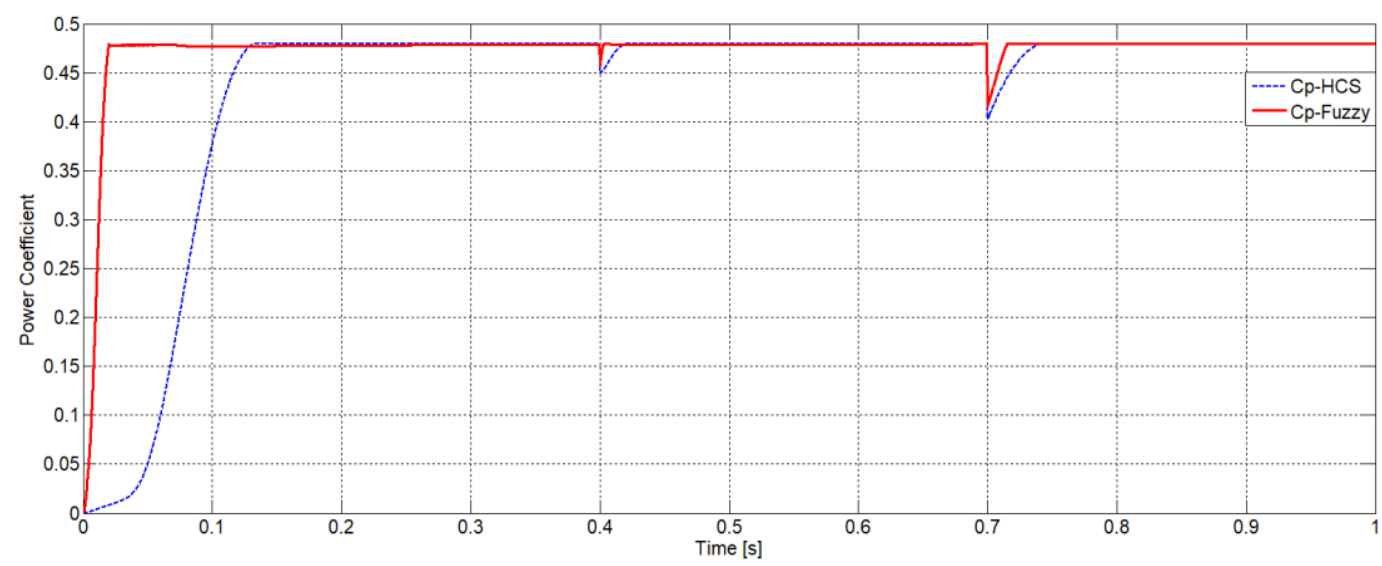

Figure 10. The power coefficient

The generator speed (or the turbine speed) is shown in Figure 11. The Fuzzy controller has a fast response time and gives a larger value than the HCS controller. Therefore, the optimum speeds of the two controllers are different. The oscillation of the Fuzzy controller is also less than that of the HCS controller. Finally, we realize that the turbine power response of the Fuzzy controller is also better than that of the HCS controller as shown in Figure 12.

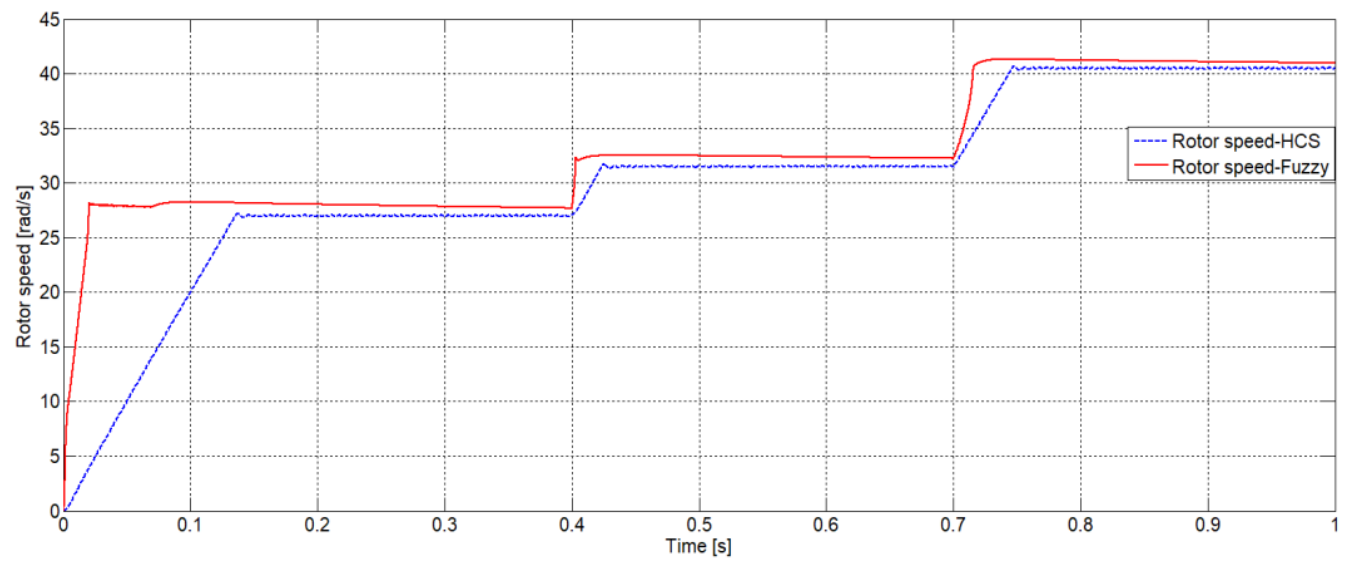

Figure 11. The rotor speed

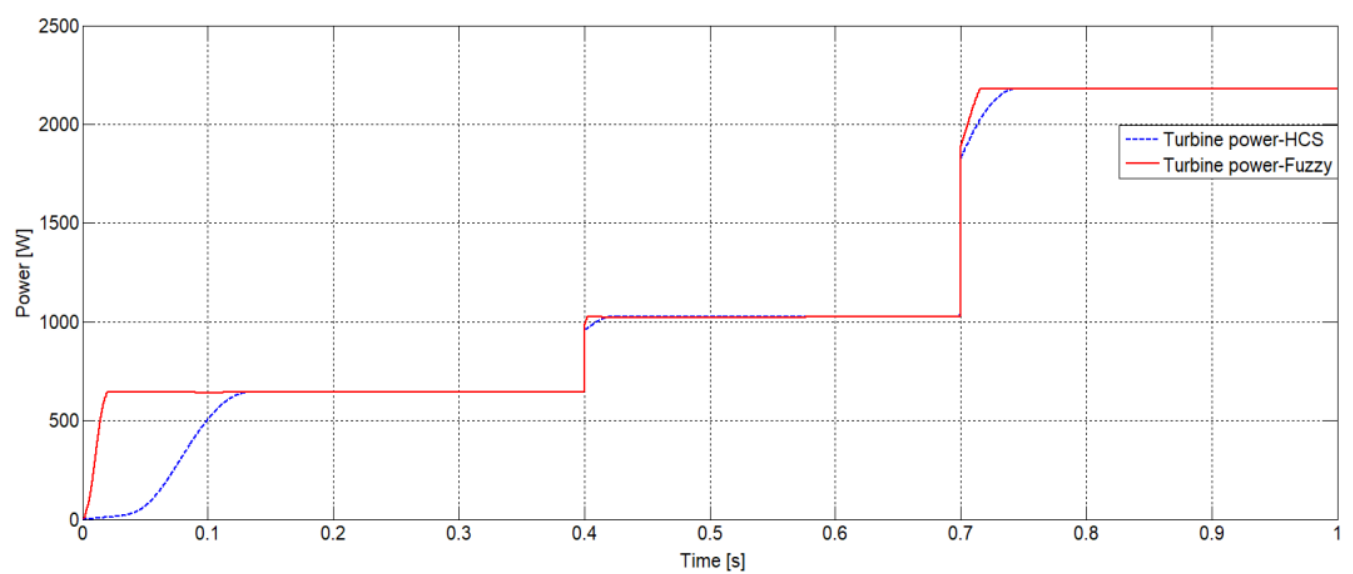

Figure 12. Turbine power 


\section{CONCLUSION}

The authors have built successfully the MPPT-Fuzzy control system. A comparison between the traditional controller (HCS) and the Fuzzy controller has proven the advantages of the Fuzzy controller. Therefore, the MPPT-Fuzzy controller is completely feasible and highly applicable to the SSWT system. The paper also proposed the control structure for SSWT. With this type of structure, the system responds is better because it reflects the actual wind speed at the control time, and the power in the range of low wind speed is exploited with the optimal value.

\section{REFERENCES}

[1] Mohamed N., et al., "Robust coordinated control using backstepping of flywheel energy storage system and DFIG for power smoothing in wind power plants," International Journal of Power Electronics and Drive Systems (IJPEDS), vol. 10, no. 2), pp. 1110-1122, 2019.

[2] Soumia K., et al., "Study of automatic generation control in two area power system with DFIG-based wind energy conversion," Journal of Power Electronics and Drive Systems (IJPEDS), vol. 10, no. 4, pp. 2118-2125, 2019.

[3] Issam M., et al., "Control of the photoelectric generator for used in feeding of the independent wind turbine system," Journal of Power Electronics and Drive Systems (IJPEDS), vol. 10, no. 3, pp. 1613-1627, 2019.

[4] Yasmine I., et al., "Improved wind system using non-linear power control," Indonesian Journal of Electrical Engineering and Computer Science, vol. 14, no. 3, pp.1148-1158, 2019.

[5] Yaakoubi, A. E., et al., "Non-linear and intelligent maximum power point tracking strategies for small size wind turbines: Performance analysis and comparison," Energy Reports, vol. 5, pp. 545-554, 2019.

[6] Rocha, P.A.C, et al., "The effects of blade pitch angle on the performance of small-scale wind turbine in urban environments," Energy, vol. 148, pp. 169-178, 2018.

[7] Tummala, A., et al., "A review on small scale wind turbines," Renewable and Sustainable Energy Reviews, vol. 56, pp. 1351-1371, 2016.

[8] Daili, Y., et al, "Implementation of a new maximum power point tracking control strategy for small wind energy conversion systems without mechanical sensors," Energy Conversion and Management, vol. 97, pp. 298-306, 2015.

[9] Kumar, D., Kalyan C., "A review of conventional and advanced MPPT algorithms for wind energy systems," Renewable and sustainable energy reviews, vol. 55, pp. 957-970, 2016.

[10] Yin, M., et al., "Aerodynamic optimization for variable-speed wind turbines based on wind energy capture efficiency," Applied Energy, vol. 221, pp. 508-521, 2018.

[11] Chang, T-P, et al., "Comparative analysis on power curve models of wind turbine generator in estimating capacity factor," Energy, vol. 73, pp. 88-95, 2014.

[12] Ganjefar, S., et al., "Improving efficiency of two-type maximum power point tracking methods of tip-speed ratio and optimum torque in wind turbine system using a quantum neural network," Energy, vol. 67, pp. 444-453, 2014.

[13] Dahbi, A., et al., "Realization and control of a wind turbine connected to the grid by using PMSG," Energy Conversion and Management, vol. 84, pp. 346-353, 2014.

[14] Kazmi, S. M. R., et al., "A novel algorithm for fast and efficient speed-sensorless maximum power point tracking in wind energy conversion systems," IEEE Transactions on Industrial Electronics, vol. 58, no. 1, pp. 29-36, 2010.

[15] Liu, J., et al., "A novel MPPT method for enhancing energy conversion efficiency taking power smoothing into account," Energy Conversion and Management, vol. 101, pp. 738-748, 2015.

[16] Abdeddaim, S., and A. Betka, "Optimal tracking and robust power control of the DFIG wind turbine," International Journal of Electrical Power and Energy Systems, vol. 49, pp. 234-242, 2013.

[17] Mousa, H. H. H, et al., "Adaptive P\&O MPPT algorithm-based wind generation system using realistic wind fluctuations," International Journal of Electrical Power and Energy Systems, vol. 112, pp. 294-308, 2019.

[18] Messalti, S, et al., "A new variable step size neural networks MPPT controller: Review, simulation and hardware implementation," Renewable and Sustainable Energy Reviews, vol. 68, pp. 221-233, 2017.

[19] Lee, C-Y, et al., "Maximum power point tracking (MPPT) system of small wind power generator using RBFNN approach," Expert Systems with Applications, vol. 38, no. 10, pp. 12058-12065, 2011.

[20] Jaramillo-L., et al., "A novel online training neural network-based algorithm for wind speed estimation and adaptive control of PMSG wind turbine system for maximum power extraction," Renewable Energy, vol. 86, pp. 38-48, 2016.

[21] Belmokhtar, K., et al., "Novel fuzzy logic based sensorless maximum power point tracking strategy for wind turbine systems driven DFIG (doubly-fed induction generator)," Energy, vol. 76, pp. 679-693, 2014.

[22] Mesemanolis, A., and Mademlis, C., "Combined maximum power point and yaw control strategy for a horizontal axis wind turbine," In 2014 International Conference on Electrical Machines IEEE, pp. 1704-1710, 2014.

[23] Chen, L., et al., "Comparison of AC/DC converters and the principles of a new control strategy in small-scale wind turbine systems," In 2012 22nd Australasian Universities Power Engineering Conference (AUPEC) IEEE, pp. 1-6, 2012.

[24] Chen, L., et al., "Multi-mode control strategy in small-scale wind turbine generators for wider operating speed range and higher efficiency operation," In 2013 IEEE Energy Conversion Congress and Exposition IEEE, pp. 3146-3153, 2013.

[25] Abdullah, M. A., et al, "A review of maximum power point tracking algorithms for wind energy systems," Renewable and sustainable energy reviews, vol. 16, no. 5, pp. 3220-3227, 2012. 